\title{
Evaluation of some Rhizobacteria as Induce Systemic Resistance or Bio-Control Agents in Controlling Root-knot Nematode, Meloidogyne incognita on Tomato
}

\author{
A.A. Anter ${ }^{\star}$, A.W. Amin ${ }^{\star}$, A. H. Ashoub** and A.S. El-Nuby ${ }^{\star *}$ \\ ${ }^{*}$ Nematology and Agriculture Zoology Department, Fac. Agric., Cairo University \\ ${ }^{* *}$ Nematology Unit, Plant Protection Department, Desert Research Center, Cairo. \\ Corresponding author email: aminamin280@gmail.com
}

\begin{abstract}
Ten bacterial strains, selected from a bulk of isolates recovered from tomato rhizosphere, were tested for their ability to induce systemic resistance or bio-control agents against Meloidogyne incognita in tomato under greenhouse condition. Results showed that all ten tested bacterial strains showed significant reduction in nematode development and reproduction. The most effective strains were Methylomonas methanica, Bacillus cereus, Bacillus brevis and Obesumbacterium proteus. They were achieving the highest reduction in nematode total population and fecundity. Plant growth was improved as a result of application of rhizobacteria. Antioxidant enzymes activity for both peroxidase and polyphenol oxidase were elevated in bacteriazied plants compared nematode infected plant as well as total phenol content. Results revealed that crude culture suspension of bacteria was more effective for reducing nematode population followed by cell-free culture filtrates, bacterial live cells and bacterial dead cells suspension, sequentially. It was concluded that these bacteria able to suppress $M$. incognita as resistance inducers for tomato plants or bio-control agents.
\end{abstract}

Keywords: Rhizobacteria, Meloidogyne incognita, induce systemic resistance, biological control, tomato.

\section{Introduction}

The root-knot nematodes, Meloidogyne spp. are one of the most economically important pest causing severe damages to a wide variety of crops particularly to tomato. Various techniques, including crop rotation, planting of resistant cultivars, and nematicides application have been used for the management of this nematode. Since the rhizosphere provides the first line of defense for roots against nematode attack, it is generally considered that rhizosphere bacteria are ideal bio-control agents. Their ability to multiply and spread in the rhizosphere environment, to colonize potential infection-sites on the root and possibly to act by direct contact with the parasites are characteristics that make them useful agents for nematode management. Studies on a number of plantmicrobe interactions showed that such antagonistic rhizobacteria can function directly by competition and antibiosis (Buchenauer, 1998). Also, indirectly by 
inducing systemic resistance (ISR) in the plant toward soil-borne pathogens. Hasky-Gunther et al. (1998) were the first who demonstrate induced systemic resistance mechanism of action by Rhizobacteria against nematode. Fatherly, Reitz et al., (2000) and Siddiqui and Shaukat, (2002\&2004) confirmed occurring of ISR by rhizobacteria.

This investigation was done to evaluate the ability of some rhizobaterial strains as ISR or bio-control agent toward root-knot nematode, Meloidogyne incognita and to exploring the performance of different bacterial component as elicitors for plant resistance and impact on plant growth.

\section{Materials and Methods}

Out of 35 bacterial strains were isolated from the rhizosphere of tomato plants only 10 isolates were consider as plant inducer after in vitro and in vivo screenings on tomato plant infested with Meloidogyne incognita. These isolates were identified as: Bacillus brevis, Bacillus cereus, Bacillus firmus, Klebsiella planticolla, Lactobacillus agilis, Lactobacillus fermentum, Methylomonas methanica, Neisseria elongata, Obesumbacterium proteus and Pseudomonas aeruginosa.

Single egg-mass culture of Meloidogyne incognita was mass rearing in tomato plants cultivated in disinfected soil and growing in greenhouse conditions. Tomato plants cv. Castel rock cultivated in $25 \mathrm{~cm}$ diam. earthen pots filled with about one kg sterilized soil ( 3 sand: 1clay V:V) were used in all experiments.

Four-weeks old tomato seedlings cv. Castol rock susceptible to Meloidogyne incognita were transplanted in the pots kept in the greenhouse at $30 \pm 5^{\circ} \mathrm{C}$ for use in the experiments. Plants were fertilized with compound fertilizer and watered as needed.

Bacterial suspensions were added as soil drench $\left(100 \mathrm{ml} /\right.$ pot at $\left.10^{9} \mathrm{CFU} / \mathrm{ml}\right)$ two days before nematode inoculation with $1000 \mathrm{~J}_{2}$ of $M$. incognita per pot. The plants under greenhouse conditions were irrigated and fertilized according to the recommendations of the Egyptians Ministry of Agriculture. The treatments were replicated four times (4 pots) in a completely randomized block design. Later, after forty five days of nematode inoculation, plants were carefully uprooted and nematodes in soil and roots were counted and recorded based on No of galls, No. of juveniles in soil, developmental stages, mature female, egg-masses numbers per plant and average eggs per egg-mass were recorded (average 10 egg-masses). The plants weights and lengths were registered. Also, peroxidase and polyphenol oxidase activity and total phenols were estimated in roots. Total soluble phenols were determined by using Folin and Ciocalteu's Phenol Reagent (Daniel and George, 1972).

Enzymes extraction form rhizobateria-treated and nematode infected roots, nematode infected roots only and healthy one were collected 7 days after nematode 
inoculation to estimate enzyme activity. Enzyme extract were prepared according to Maxwell and Batemen (1967). Assay of peroxidase activity (POX), changes in POX activity were determined following the procedure described by Sridhar and Ou (1974). POX activity was expressed as change in absorbance ( $\triangle$ O.D $470 \mathrm{~nm}$ ) per min/gram fresh weight. Assay of polyphenol oxidase activity (PPO), changes in PPO activity were determined according to Maxwell and Batemen (1967). The activity of PPO was expressed as ( $\triangle$ O.D $495 \mathrm{~nm}$ ) $/ 1.0 \mathrm{ml}$ of extract per min per gram fresh weight.

Crude culture suspension (CS), cell free filtrate (F), viable or life cell (LCS) suspension and heat-killed cell suspension (DCS) of the most vigorous four bacteria: Bacillus brevis, Bacillus cereus, Methylomonas methanica and Obesumbacterium proteus were evaluated separately for their ability to suppress $M$. incognita severity and induced systemic resistance in tomato plants against nematode. Four-week old tomato seedlings were transplanted in disinfected earthen pots. Different bacterial concertinos were adjusted at $10^{9} \mathrm{CFU} / \mathrm{ml}$ for CS, LCS and DCS. All forms were added to soil $(100 \mathrm{ml} / \mathrm{pot})$ two days before nematode inoculation with $1000 \mathrm{M}$. incognita $\left(\mathrm{J}_{2 \mathrm{~s}}\right)$ per plant. Pots were kept in a greenhouse for 45 days then plants were uprooted and nematode criteria were recorded.

The data were subjected to analysis of variance and means were separated by the least significant difference LSD at $(p=0.05)$ using PLABSTAT program Version 3, Institute fuer Pflanzen zuechtung, Universitaet Hohenheim.

\section{Results}

The results in Table 1 conclude that all selected strains could arrest $M$. incognita reproduction and development compared to untreated control. The most effective isolate was $M$. methanica where it impaired the different nematode stages and total population and its fecundity (eggs/egg-mass). M.methanica gave 97 galls and 15 egg-masses/plant compared to untreated control which recorded 678 galls and 201 egg-masses. Its effect was continued to diminish developmental stages (DS) to 75 and mature females (MF) to 17 compared to 675 (DS) and 239 (MF) in untreated control. Consequently, the total population recorded 260 individuals compared to 3824 in untreated control. This bacterium could inhibit $M$. incognita fecundity (112) while untreated control favored nematode fecundity to 640 eggs/egg-mass. However, the most effective three strains followed $M$. methanica in suppression nematode total population were $B$. cereus, $O$. proteus and $B$. brevis followed by $B$. firmus and $P$.aeruginosa. On the other hand, the weakest isolate was $N$. elongata where recorded 1444 as total population.

Effect of rhizobacterial strains on plant growth presented in Table 2 showed that all bacterial strains enhanced tomato growth compared to nematode infected plants. Generally, all treatments exhibited an increment in total plant weight and length compared by untreated control. The minimum impact on growth criteria was recorded by both $K$. planticolla and $N$. elongata. The same trend was observed on plant length. 
Table (1).Effect of some bacterial strains on development and reproduction of Meloidogyne incognita infected tomato plants under greenhouse conditions.

\begin{tabular}{|c|c|c|c|c|c|c|c|c|c|}
\hline Bacterial strains & Galls & $\begin{array}{c}\text { Juveniles } \\
\text { in soil }\end{array}$ & $\begin{array}{c}\text { Number of } \\
\text { Develop- } \\
\text { mental stages }\end{array}$ & Females & $\begin{array}{c}\text { Egg- } \\
\text { masses }\end{array}$ & $\begin{array}{c}\text { Total } \\
\text { population }\end{array}$ & $9 \mathrm{R}^{*}$ & $R f^{* t}$ & $\begin{array}{c}\text { Number of } \\
\text { Eggs/ } \\
\text { egg- mass }\end{array}$ \\
\hline Bacillus brevis & 94 & 316 & 112 & 44 & 39 & 472 & 87.7 & 0.472 & 210 \\
\hline Bacillus cereus & 116 & 280 & 90 & 41 & 38 & 411 & 89.3 & 0.411 & 138 \\
\hline Bacillus firmus & 281 & 650 & 209 & 50 & 41 & 909 & 762 & 0.909 & 239 \\
\hline Websiella planticolla & 191 & 948 & 164 & 96 & 93 & 1208 & 68.4 & 1.208 & 305 \\
\hline Lactobacillus agilis & 100 & 768 & 223 & 62 & 57 & 1051 & 72.5 & 1.051 & 231 \\
\hline Lactobacillus fermentum & 385 & 760 & 416 & 111 & 96 & 1287 & 66.3 & 1.287 & 319 \\
\hline Methyiomonas methanica & 97 & 168 & 75 & 17 & 15 & 280 & 932 & 0.280 & 112 \\
\hline Neisseria elongata & 398 & 773 & 594 & 77 & 73 & 1444 & 62.2 & 1.444 & 517 \\
\hline Obesumbacterium proteus & 147 & 283 & 143 & 43 & 40 & 469 & 87.7 & 0.469 & 182 \\
\hline Pseudomonas aeruginosa & 339 & 633 & 319 & 82 & 69 & 1034 & 73.0 & 1.034 & 285 \\
\hline Control & 678 & 2910 & 675 & 239 & 201 & 3824 & - & 3.824 & 640 \\
\hline LSD 0.05 & 9.0 & 120.0 & 20.8 & 9.6 & 9.6 & - & - & - & 18.9 \\
\hline
\end{tabular}

w/6R- Total population Reduction, -Fit- Rep noduction factor 
Table (2). Effect of some bacterial strains on growth parameters of tomato plants infected with Meloidogyne incognita under greenhouse conditions.

\begin{tabular}{|c|c|c|c|c|c|c|}
\hline Bacterial stra ins & $\begin{array}{c}\text { Fresh shoot } \\
\text { weight }\end{array}$ & $\begin{array}{c}\text { Fresh root } \\
\text { weight }\end{array}$ & $\begin{array}{l}\text { Shoot } \\
\text { length }\end{array}$ & $\begin{array}{l}\text { Root } \\
\text { length }\end{array}$ & $\begin{array}{c}\% / \\
\text { plant weight } \\
\text { increment }\end{array}$ & $\begin{array}{l}\text { \% } \\
\text { plant length } \\
\text { increment }\end{array}$ \\
\hline Bacillus brevis & 32.6 & 12.1 & 51 & 34 & 22.9 & 14.1 \\
\hline Bacillus cereus & 35.2 & 14.5 & 52 & 35 & 30.5 & 16.1 \\
\hline Bacillus firmus & 33.4 & 13.8 & 51 & 34 & 28.9 & 14.1 \\
\hline Websiella planticolla & 29.4 & 10.3 & 47 & 30 & 132 & 52 \\
\hline Lactobacillus agilis & 30.2 & 11.2 & 50 & 34 & 16.7 & 13.1 \\
\hline Lactobacillus fermentum & 31.5 & 11.3 & 51 & 33 & 19.4 & 13.1 \\
\hline Methylomonas methanica & 34.8 & 13.3 & 54 & 38 & 28.2 & 20.7 \\
\hline Neisseria el ongata & 27.2 & 11.0 & 48 & 32 & 9.6 & 8.8 \\
\hline Obesumbecterium proteus & 32.4 & 12.4 & 50 & 36 & 23.1 & 15.1 \\
\hline Pseudomonas aenuginosa & 32.1 & 11.6 & 49 & 32 & 21.0 & 9.9 \\
\hline Untreated & 25.1 & 9.4 & 44 & 29 & - & - \\
\hline LSD 0.05 & 2.3 & 1.6 & 3.3 & 3.5 & - & - \\
\hline
\end{tabular}


Data in Table 3 revealed that the activity of certain biological processes was enhanced as a result of using bacterial strains which considered as inducers for the systemic resistance of tomato plants and bio-control agents on nematode. The presence of $M$. incognita only and without any interference led to increase the total phenols (19.6 $\mu \mathrm{g} / \mathrm{g} . \mathrm{fwt})$ compared to untreated and uninfected plant (healthy plant) which recorded $16.4 \mu \mathrm{g} / \mathrm{g}$.fwt. On the other hand, the tomato plants treated with different selected bacterial strains showed increment in their total phenols. The highest value was related to $M$. methanica $(27.1 \mu \mathrm{g} / \mathrm{g}$.fwt) followed by $B$. cereus, B.brevis, O. proteus and $B$. firmus. They recorded 26.3, 26.6, 25.5 and 24.2 $\mu \mathrm{g} / \mathrm{g}$.fwt respectively. The lowest value was registered by $N$. elongata. $(20.5$ $\mu g / g . f w t)$.

Table (3). Effect of some bacterial strains on peroxidase, polyphenol oxidase activities and total phenol content in tomato roots infected with Meloidogyne incognita.

\begin{tabular}{|c|c|c|c|c|c|}
\hline \multirow{3}{*}{ Bacterial strains } & \multirow{3}{*}{$\begin{array}{c}\text { Total } \\
\text { phenols } \\
\mu \mathrm{g} / \mathrm{g} \text { f wt }\end{array}$} & \multicolumn{4}{|c|}{ Enzymes } \\
\hline & & \multicolumn{2}{|c|}{ Peroxidase } & \multicolumn{2}{|c|}{ Polyphenol oxidase } \\
\hline & & Activity & $\begin{array}{l}\text { Relative } \\
\text { activity }\end{array}$ & Activity & $\begin{array}{c}\text { Relative } \\
\text { activity }\end{array}$ \\
\hline Bacillus brevis & 26.3 & 2.2 & 1021.9 & 0.44 & 1031.6 \\
\hline Bacillus cereus & 26.6 & 2.3 & 1062.6 & 0.55 & 1262.5 \\
\hline Bacillus firmus & 24.2 & 2.1 & 979.7 & 0.43 & 985.5 \\
\hline Klebsiella planticolla & 22.2 & 1.9 & 876.4 & 0.20 & 454.2 \\
\hline Lactobacillus agilis & 22.8 & 1.9 & 903.0 & 0.34 & 777.5 \\
\hline Lactobacillus fermentum & 21.2 & 1.8 & 826.3 & 0.32 & 746.7 \\
\hline Methylomonas methanica & 27.1 & 2.3 & 1073.6 & 0.57 & 1308.7 \\
\hline Neisseria elongata & 20.5 & 1.7 & 807.5 & 0.30 & 669.8 \\
\hline Obesumbacterium proteus & 25.5 & 2.2 & 1011.0 & 0.50 & 1147.0 \\
\hline Pseudomonas aeruginosa & 22.8 & 2.0 & 924.9 & 0.42 & 962.3 \\
\hline Check (infected control) & 19.6 & 0.5 & 226.9 & 0.19 & 431.1 \\
\hline Healthy (Untreated) & 16.4 & 0.2 & 100.0 & 0.04 & 100.0 \\
\hline LSD 0.05 & 0.7 & 0.4 & - & 0.06 & - \\
\hline
\end{tabular}

Peroxidase (POX) and polyphenol oxidase (PPO) were increased by all bacterial strains treatments (Table 3 ). The maximum POX activity was induced by M. methanica (2.3 mg/g.fwt). No significant in the POX activity was recorded by the rested bacterial strains. On the other side, the nematode infected plants exhibited enzyme activity $(0.5 \mathrm{mg} / \mathrm{g}$.fwt $)$ higher than healthy one $(0.2 \mathrm{mg} / \mathrm{g}$.fwt) which was the lowest value.

Enzyme activity of PPO indicated that $M$. methanicahas the highest between all treatments followed by B.cereus> O.proteus $>$ B.brevis $>$ B.firmus $>$ P.aeruginosa $>$ L.agilis> L.fermentum $>$ N.elongata $>$ K.planticolla. The enzyme activity of infected plants remained higher than the healthy one that rested at the lowest value.

Results presented in Table 4 noted that all bacterial components were effective on suppressing nematode development and reproduction. All treatments 
achieved high reduction in number of galls was related to the crude suspensions followed by filtrates. The lowest reduction in galls was exhibited by the dead cells suspensions. The less effective strain was B. brevis (44.5\%). Egg-masses production was highly depressed by crude suspension of all strains. The most effective strain was $M$. methanica which registered $96.2 \%$ reduction. The rested bacterial strains were arranged as follows: B. cereus, $O$. proteus and B. brevis. Similarly, filtrate additions keep their efficiency as previously ranked. Viable and dead cell suspensions were less effective than $(C S)$ or $(F)$ in reducing eggmasses/plant. The reduction in nematode total population was related to crude form, values of $M$. methanica $(90.4 \%)$, B. cereus (86.5\%), O. Proteus (85\%) and B. brevis (84.5\%).

Due to the No. of eggs/egg-mass $M$. methanica was the most successful strain could reduce the reproductive potency of $M$. incognita $(74.3 \%)$ followed by $B$. cereus $(70.9 \%)$, B. brevis (62.8) and O. proteus (57.6). The lowest effect was done by $B$. brevis (31.3\%) as (DCS). The rates of build-up take the same trend, while CS was the most suppresser for nematode reproduction (Rf) in all tested bacteria (Fig. $1)$.

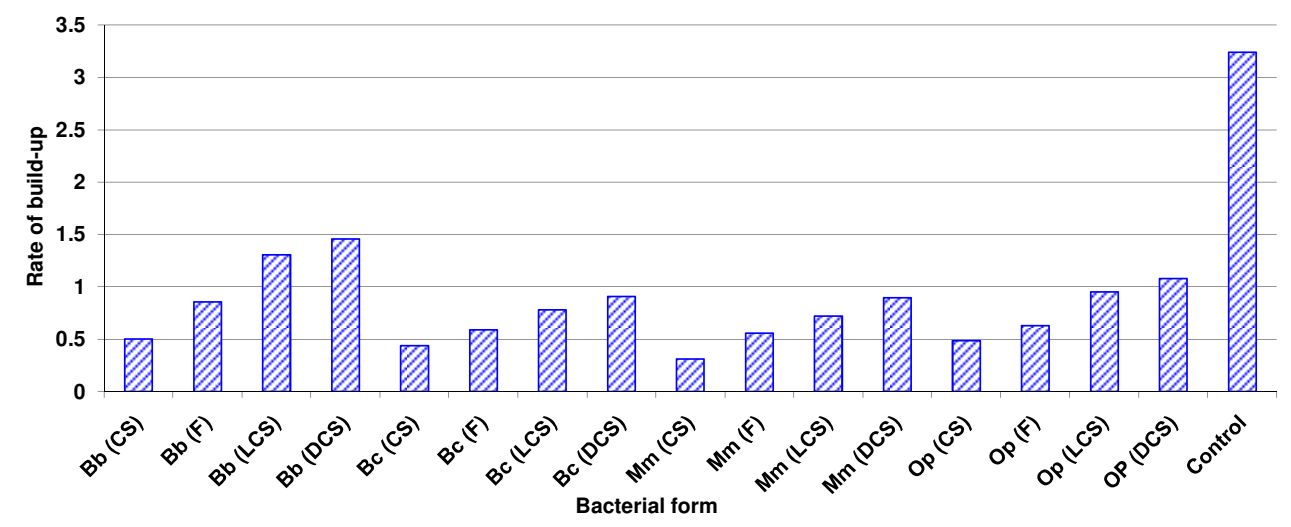

Fig. (1). Effect of some bacterial strains applied as crude suspension, culture filtrate, live and killed cells on Meloidogyne incognita reproduction infected tomato plants cv. Castle rock under greenhouse conditions.

$\mathrm{CS}=$ Crude suspension, $\mathrm{F}=$ Filtrate, $\mathrm{LCS}=\mathrm{Live}$ cell, $\mathrm{DSC}=\mathrm{Dead}$ cell, $\mathrm{Bb}=$ Bacillus brevis, $\mathrm{Bc}=$ Bacillus cereus, $\mathrm{Mm}=$ Methylomonas methanica, $\mathrm{Op}=$ Obesumbacterium proteus

Results in Table 5 pointed to the different forms of all bacterial strains and plant growth criteria. The most effective strain was M. methanica as (CS), which exhibited the maximum improvement for shoot and root fresh weight besides shoot dry weight which recorded 30.6, 6.3 and $4.4 \mathrm{gm}$. respectively. Dead cells of $M$. methanica had the priority than other strains. 
Table (4). Effect of some bacterial strins applied as cuude suspension, culture filtrate, live and killed cells on development and reproduction of Meloidogyne incognita infected tomato plants under greenhouse conditions.

\begin{tabular}{|c|c|c|c|c|c|c|c|c|c|c|c|c|c|c|c|}
\hline \multirow[b]{2}{*}{$\begin{array}{l}\text { Ductende } \\
\text { serand }\end{array}$} & \multirow[b]{2}{*}{ Dudewestann (a.r) } & \multirow{2}{*}{ No ot } & \multirow[b]{2}{*}{ sin } & \multirow{2}{*}{ Noot } & \multirow[b]{2}{*}{$\mathrm{sin}$} & \multirow{2}{*}{$\begin{array}{l}\text { Noot } \\
\text {-ses: } \\
\text { max: }\end{array}$} & \multirow[b]{2}{*}{ su } & \multirow{2}{*}{ 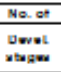 } & \multirow[b]{2}{*}{$x$} & \multirow{2}{*}{ No.at } & \multirow[b]{2}{*}{$\mathbf{s i n}$} & \multirow{2}{*}{$\frac{\text { Iotal }}{\text { Propulation }}$} & \multirow[b]{2}{*}{ sis } & \multirow{2}{*}{ 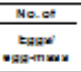 } & \multirow[b]{2}{*}{$\mathrm{sin}$} \\
\hline & & & & & & & & & & & & & & & \\
\hline & 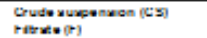 & $\begin{array}{l}82 \\
180\end{array}$ & $\begin{array}{l}2.8 \\
6.2\end{array}$ & $\begin{array}{l}280 \\
222\end{array}$ & $\begin{array}{ll}841 \\
\text { TeI }\end{array}$ & 4 & $\begin{array}{l}30.8 \\
\text { Te. }\end{array}$ & $\begin{array}{l}110 \\
201\end{array}$ & $\begin{array}{l}39.9 \\
\text { a.g }\end{array}$ & $a$ & $\pi s .2$ & $\begin{array}{l}502 \\
094\end{array}$ & 849 & 28 & 622 \\
\hline \multirow[t]{5}{*}{$\cos$} & 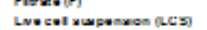 & 272 & 4.1 & $\begin{array}{l}221 \\
290\end{array}$ & 01.4 & $T$ & 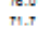 & $\begin{array}{l}2 \mathrm{a} \\
2 \mathrm{Te}\end{array}$ & 4.4 & $s$ & T.8. & 300 & 927 & nis & 249 \\
\hline & Dowd cet wapeneon (DC:S) & $28 z$ & 4.9 & sto & 002 & $\approx$ & o. & 40 & 2.3 & 112 & $\mathrm{e} .2$ & 49 & 920 & 280 & 212 \\
\hline & Untrensed interised plitest & 927 & - & 2302 & - & 278 & - & $T z$ & - & 108 & - & 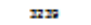 & - & 908 & - \\
\hline & Mowe sxatsen & 2773 & - & 22 & - & 112.4 & & 220.8 & - & 1224 & - & - & - & 316.4 & - \\
\hline & Coudexusenveron & 84 & s.s. & $2 n$ & $8: 0$ & $n z$ & s.s & 2 & $=5.8$ & 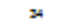 & $m s$ & דם & ses & 48 & Tos \\
\hline \multirow{5}{*}{ 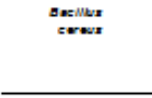 } & peres: & 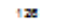 & те. & es: & 728 & $\sqrt{2}$ & $=0.0$ & тe & $=$ & $a$ & $=8$ & 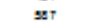 & 818 & 180 & 62. \\
\hline & Lvecel xugeneren & 141 & T. & eno & $T 24$ & $\theta$ & 79.8 & so & $=0.0$ & $\theta$ & $\pi \cdot$. & $\pi \mathrm{s}$ & Tes & $m$ & 942 \\
\hline & Dewd extil wapenesen & 242 & $=0.0$ & 718 & 67.4 & 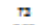 & 74.9 & 100 & as. & $=2$ & T2. 4 & ne & 720 & $\mathrm{se} 4$ & $48:$ \\
\hline & 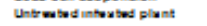 & דנפ & - & 2302 & - & $2 \pi$ & - & $t=$ & - & 208 & - & $22 \pi$ & - & 900 & - \\
\hline & Man sxitand & $2: 3$ & . & 8อ2. & . & 988 & . & 2128 & . & 1073 & . & & & 고요 & \\
\hline \multirow{7}{*}{$\begin{array}{l}\text { Mothplamamax } \\
\text { methanisa }\end{array}$} & Coude xusermeson & 6 & 57.8 & 212 & 524 & 11 & $\mathrm{x.2}$ & 80 & s.t. & 17 & 2.8 & 210 & 804 & 19 & 742 \\
\hline & rese: & se & a.s & S9 & 722 & 27 & $n .2$ & as & n.e & $n$ & 0.4 & $=7$ & $82:$ & 48 & Tos \\
\hline & 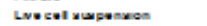 & 102 & T. & $=2$ & 748 & 50 & 52.0 & 117 & a.s & $s 2$ & a.2 & 721 & $\pi T . T$ & 200 & $9+2$ \\
\hline & 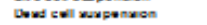 & 202 & en: & בות & er.e & $s$ & T. & 122 & a. & 60 & 00.9 & $m$ & 724 & $2=1$ & 92.7 \\
\hline & 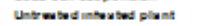 & 927 & - & 2302 & - & $2 \pi$ & - & 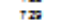 & - & 208 & - & $m$ & - & 9 & . \\
\hline & Mase tuxsena & 2012 & . & צנו & . & 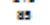 & : & 2128 & . & 24 & . & $\because$ & . & 291.8 & . \\
\hline & Coude xusenenes & 24 & $=8$ & $m$ & 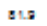 & $y_{4}$ & 87.8 & 92 & g2. 7 & 28 & s.s. & ses & 820 & 210 & פו \\
\hline \multirow{5}{*}{ Obenumbectoutum } & reve: & 62 & 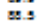 & 92 & Tas & $=$ & T. & 9 & n. & $a$ & Tas & $\mathrm{es}$ & 800 & me & T. T⿱ \\
\hline & Lvecel xusenteren & 118 & $\mathrm{Te} .1$ & $\mathrm{TgO}$ & eas & 72 & 74.2 & $s$ & 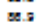 & 74 & Te. 0 & M & Te. T & 201 & 928 \\
\hline & Dewd ext wapenenen & 187 & es. & se & 62: & n & et. 4 & 100 & 78.0 & $\mathrm{n}$ & e.t & 1078 & set & 2rt & 498 \\
\hline & Untroned ntwatsod plant & דיבפ & - & 2302 & - & $2 \pi$ & - & $t z$ & - & 208 & - & $n z$ & - & 90 & - \\
\hline & Maxn Bxisten & $m \leq 2$ & . & 943.4 & . & ing & . & 218.4 & . & 114.4 & . & - & . & 2783.4 & - \\
\hline \multicolumn{2}{|c|}{ Men Excturwi Tann (CS) } & 703 & $\overline{-}$ & 327.8 & - & 223 & . & 740 & - & 32y & - & - & - & 171.9 & - \\
\hline \multicolumn{2}{|c|}{ 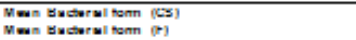 } & 1120 & . & 480 & . & $48 y$ & . & 1128 & . & 9 & . & . & . & 1009 & . \\
\hline Man textarul tomn & (Les) & 1879 & . & Tus 0 & . & 87.3 & . & 187.0 & . & axy & . & . & . & 254.8 & - \\
\hline \multicolumn{2}{|c|}{ 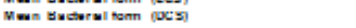 } & zaz: & . & $781 . x$ & - & 720 & . & 2128 & - & 87.0 & . & . & - & 2850 & - \\
\hline \multicolumn{2}{|c|}{ Lsuoug tuxtana (a) } & 22.1 & & 203 & & $9 x$ & & 8.2 & & 8 & & & & 17.0 & \\
\hline \multirow{2}{*}{\multicolumn{2}{|c|}{ 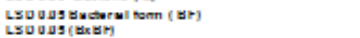 }} & 200 & & 208 & & 2. & & 13,5 & & 22 & & & & & \\
\hline & & 448 & & 40.8 & & & & 304 & & 20.9 & & & & 920 & \\
\hline
\end{tabular}


Table (5). Effect of some bacterial strains applied as orude suspension, filtrate, live and killed cells on growth parameters of tomato infected by Meloidogyne incognita under greenhouse conditions.

\begin{tabular}{|c|c|c|c|c|c|c|c|c|c|c|c|}
\hline Bacteria strains & Form & $\begin{array}{c}\text { Fresh shoot } \\
\text { wel ght }\end{array}$ & $\% *$ & $\begin{array}{c}\text { Dry shoot } \\
\text { weight }\end{array}$ & $\%$ & $\begin{array}{l}\text { Shoot } \\
\text { langth }\end{array}$ & $\%$ & $\begin{array}{c}\text { Root } \\
\text { wel ght }\end{array}$ & $\% 1$ & $\begin{array}{c}\text { Root } \\
\text { lingth }\end{array}$ & $\%$ \\
\hline & Gude 5 Ls pensil on & 27.4 & 242 & 3.8 & 14.2 & 51.7 & 24.5 & 5.5 & 25.3 & 350 & 18.1 \\
\hline Bacillus & Filtrate & 26.7 & 223 & 3.6 & 99 & 45.3 & 140 & 5.3 & 220 & 340 & 15.7 \\
\hline \multirow[t]{4}{*}{ brevis } & $\begin{array}{l}\text { Live coll suspension } \\
\text { Dead coll } 5 \text { us pensilon }\end{array}$ & $\begin{array}{l}25.4 \\
24.2\end{array}$ & $\begin{array}{l}18.2 \\
139\end{array}$ & $\begin{array}{l}3.6 \\
3.4\end{array}$ & $\begin{array}{l}9.6 \\
3.2\end{array}$ & $\begin{array}{l}45.3 \\
40.3\end{array}$ & $\begin{array}{c}140 \\
3.3\end{array}$ & $\begin{array}{l}4.6 \\
4.6\end{array}$ & $\begin{array}{l}10.7 \\
10.2\end{array}$ & $\begin{array}{l}30.3 \\
29.7\end{array}$ & $\begin{array}{l}5 . \\
3.4\end{array}$ \\
\hline & Untrea ted infes to d plant & 20.8 & - & 3.3 & - & 390 & - & 4.12 & - & 28.7 & - \\
\hline & Mean Bacteria & 24.9 & - & 3.5 & - & 44.3 & - & 4.8 & - & 31.5 & - \\
\hline & Gude 5 us pensi on(Cs) & 30.5 & 319 & 4.4 & 25.7 & 57.3 & 320 & 5.9 & 30.3 & 36.0 & 20.7 \\
\hline \multirow{6}{*}{ cereus } & Filtrate (F) & 29.0 & 28.3 & 40 & 19.3 & 50.3 & 22.5 & 5.5 & 25.3 & 34.7 & 17.3 \\
\hline & Llve call suspension (LCs) & 28.3 & 26.6 & 39 & 17.3 & 48.7 & 199 & 5.1 & 18.8 & 32.3 & 11.3 \\
\hline & Dead cell 5 us pensi on (DCs) & 26.5 & 21.6 & 3.8 & 15.4 & 47.0 & 170 & 4.9 & 15.1 & 30.7 & 6.5 \\
\hline & Untreated Intested plant & 20.8 & - & 3.3 & - & 390 & - & 4.1 & - & 28.7 & - \\
\hline & Maan Bacteria & 27.0 & - & 3.9 & - & 48.5 & - & 5.1 & - & 32.5 & - \\
\hline & Grude 5 us pensil on & 30.6 & 320 & 4.4 & 25.3 & 58.3 & 33.1 & 6.3 & 34.5 & 37.0 & 22.5 \\
\hline \multirow{6}{*}{$\begin{array}{l}\text { Medhylomonas } \\
\text { medranica }\end{array}$} & Filtrate & 29.6 & 29.7 & 4.1 & 21.2 & 510 & 23.5 & 5.5 & 25.7 & 35.0 & 18.1 \\
\hline & Llve cell suspension & 28.9 & 280 & 4.1 & 19.9 & 490 & 20.4 & 5.3 & 22.6 & 33.7 & 149 \\
\hline & Dead cell 5 us pension & 28.3 & 26.5 & 39 & 17.1 & 47.3 & 17.6 & 5.2 & 20.4 & 30.7 & 6.5 \\
\hline & Untreated Infested pla nt & 20.8 & - & 3.3 & - & 390 & - & 4.1 & - & 28.7 & - \\
\hline & Mean Bacteria & 27.6 & & 3.95 & - & 48.9 & - & 5.3 & - & - & - \\
\hline & Grude 5 us pension on & 28.9 & 280 & 40 & 18.4 & 520 & 250 & 5.6 & 26.4 & 35.7 & 19.6 \\
\hline \multirow{5}{*}{$\begin{array}{c}\text { Obesumbac zerium } \\
\text { prozeus }\end{array}$} & Filtrate & 26.2 & 20.6 & 39 & 16.2 & 47.7 & 182 & 5.3 & 22.2 & 34.3 & 16.5 \\
\hline & Live cell suspension & 22.5 & 7.5 & 3.6 & 10.0 & 43.3 & 100 & 4.7 & 12.6 & 30.7 & 6.5 \\
\hline & Dead call 6 us pension on & 21.5 & 3.1 & 3.5 & 6.1 & 40.3 & 3.3 & 4.7 & 11.4 & 30.3 & 5.5 \\
\hline & Untreated I Ine sted pla nt & 20.8 & - & 3.3 & - & 390 & - & 4.1 & - & 28.7 & - \\
\hline & Mean Bacteria & 24.0 & - & 3.6 & - & 44.5 & - & 4.9 & - & 31.0 & - \\
\hline Mean Bacterlal form (C3) & & 29.4 & $\cdot$ & 4.1 & - & 54.8 & $\cdot$ & 5.8 & $\cdot$ & 35.9 & - \\
\hline Mean Bacterlal form (F) & & 27.9 & - & 3.9 & - & 48.6 & - & 5.4 & - & 34.5 & - \\
\hline Mean Bacterlal form (LCS) & & 26.3 & - & 3.8 & - & 46.6 & - & 4.9 & - & 31.8 & - \\
\hline Mean Bacterial form (DCs) & & 25.1 & - & 3.7 & - & 43.8 & - & 4.8 & - & 30.3 & - \\
\hline LDS 0.05 Bacterla (B) & & 0.7 & & 0.4 & & 1.6 & & 0.6 & & 2.5 & \\
\hline LDS 0.05 Bacterlal form (BF) & & 1.8 & & 0.4 & & 1.8 & & 0.5 & & 1.7 & \\
\hline LDS 0.05 (BXBF) & & 3.5 & & 0,8 & & 3.7 & & 1.0 & & 3.4 & \\
\hline
\end{tabular}




\section{Discussion}

These results were in agreement with Valerie et al., (2001) where they investigated the possibility of soil-born Pseudomonas spp. and Bacillus cereus for induction resistance. They added that the application of these bacteria reduce nematodes fecundity, increase the proportions of distorted females and produced females with fewer eggs. Studies of Marleny et al., (2008) led to the hypothesis that induction of soil suppressiveness against $M$. incognita using inoculants is related to soil microbial activity and rhizosphere bacterial populations. They added that the selected microbial inoculants increase rhizosphere bacterial populations. Besides the previous effects, Vetrivelkalail et al., (2010) pointed to the nematicidal action of Pseudomonas spp., Bacillus spp. and Methylobacterium spp. against $M$. incognita. Also results present here can be clarify as a network form with complex interactions among bacteria, nematodes, plants and environment to control populations of plantparasitic nematodes in natural conditions (Kerry,2000). Rhizobacteria have many different modes of action in the soil, their effects directly through antagonizing by means of the production of toxins, lytic enzymes and other anti-nematode products (Siddiqui and Mahmood, 1999 and Giannakou et al., 2007). Also, rhizobacteriamediated induced systemic resistance-ISR- (Van Loon et al., 1998 and Ramamoorthy et al., 2001).

This plant encouragement is due to the microbial residents of the rhizosphere, those represent a potential reservoir of biological agents which can suppress nematode multiplication consequently the nematode damage diminishes. In the otherwise an induction resistance occurs within host that can decrease nematode infection. Siddiqui et al., (2007) support our results; they found that inoculation of any PGPR species alone or together with Rhizobium increased plant growth in $M$. javanica inoculated plants. Also Ali et al. (2002) stated that, soil drench with some strains of $P$. aeruginosa and Pseudomonas sp. significantly reduced populations of $M$. javanica and subsequent root-knot disease severity with enhanced protein contents and yield of mungbean plants. Similarly another investigation confirmed that $B$. cereus $\mathrm{S} 18$ is an effective bio-control agent towards M. incognita on a broad spectrum of hosts' plant. Mahdy (2002) demonstrated that all crops treated with $B$. cereus $S 18$ combined with $M$. incognita showed plant growth enhancement when compared with the bacteria untreated crops.

The mechanisms by which plant growth is improved may be similar to those exhibited by rhizosphere microorganisms and include the production of phytohormones, promotion through enhanced availability of nutrients, reduction of ethylene levels, production of antibiotics and induced systemic resistance (Holland, 1997). Suppressing nematode damage with rhizobacterial strains increased tomato root weight, which could also account for some of the observed suppression; as reducing galls, stopping revitalization root tips. This may stop their growth or cause excessive branching of roots, paving the way to normal function of roots such as 
uptake and transport water and nutrients. Positive impact extended to improve plant biomass and height, (Marleny et al., 2008).

This effective role of the total phenols was investigated since 1959 where Clark et al., related the mechanism of disease resistance to the phenolic compounds. They added that this activity due to the quinicacid or caffeic acid parts of chlorogenic acid which are released by the action of hydrolytic enzymes such as esterases. Also, certain phenolic compounds like acetylenes, terpenoid aldehydes, sesquiterpenoids and phenoxypropionic acid derivatives are known to have nematicidal activity (Veech, 1979; Mori et al., 1982; Hayashi et al., 1983). In 1985 Mahajan et al., indicated that quinones are involved in imparting nematicidal activity.

These previous results are due to the synthesis and accumulation of these enzymes which are frequently associated with plant defense against various pathogens where they are catalysts for the oxidation of substrates like phenol and its derivates by hydrogen peroxide (Buonario \& Montalbini, 1993 and Lebeda et al., 1999). The role of the peroxidase in plant defense systems is to remove the toxic effect of hydrogen peroxide from tissues and to participate in the synthesis of phenolic compounds and the building of the intermolecular bonds to fortify cell walls at the sites of pathogen invasion (Repka \& Lovakova, 1994 and Passardi et al., 2004). So peroxidase is a key enzyme in the biosynthesis of lignin (Bruce and West, 1989). Remarkable increases were observed in the peroxidase activity of all the cellular compounds, viz. soluble fraction, mitochondria and microsomes. It is previously suggested that peroxidase is an ISR marker.

These results demonstrated that efficacy of whole bacterial culture (CS) have a pronounced ability to ISR against nematode. However, (CS) represent rhizobacteria in viable state and their metabolites; such antibiotics, siderophores or/and other compounds like hormones, acids and other toxic compounds become more lethal to nematode or by another meaning (CS) gathered two advantages rerated to viable cells and metabolites so showed greater impact than other component. Several bacterial identified as ISR elicitation in different plant species as follow: lipopolysaccharides: lipid $A$; O-antigenic sidechain, siderophores: pseudobactins; pyochelin; salicylic acid (SA), flagella, antibiotics: pyocyanin, 2,4diacetylphloroglucinol $\mathrm{N}$-acylhomo serine lactones and volatile of systemic resistance in tomato plants (Van Loon and Bakker, 2005).

Live and dead cells also have the ability to induce systemic resistance. This was clearly observed by reduction of total nematode population and supported by the results of (Reitz et al., 2000) which demonstrated that living and heat-killed cells of R.etli induced potato systemic resistance against Globodera pallida infection. They suggested that heat-stable surface structures such as exopolysaccharides (EPS) and/or lipopolysaccharides (LPS) of $R$. etli G12 act as inducing agents. The 
highest effect resulted from $M$. methanica may due to it is gram-negative bacterium and may have lectin binding structures in the LPS and EPS layers of the cell wall membrane as in Pseudomonads (Lotan et al., 1975). The resistance inducing activity of bacterial metabolites to diseases has been described in literature (Schonbeck et al., 1980). Also, culture filtrate of rhizobacteria could ISR against nematode (Hasky-Günther et al., 1998). This ability may due to certain compounds including siderophores, 2,3-butanediol, the compound 2,3-butanediol that produces by Bacillus spp. and it is not only elicits ISR, but it also involved in promoting growth (Ryu et al., 2003).

Enhancement of plant growth are due to the microbial metabolites of the rhizobacteria under study which have double impact; indirectly by suppress nematode reproduction resulting in relief the adverse effect on plant fitness or directly via releasing some beneficial matters such nutrients, hormones and others which improve plant health.

Our study indicated that the potent rhizobacteria isolated and identified as Bacillus brevis, Bacillus cereus, Methylomonas methanica and Obesumbacterium proteus, could antagonistic to root-knot nematodes and could be developed into a valuable crop management tool to reduce the deleterious impact of these nematodes on plant growth. Also, enzyme activities elevation in bacterial treated roots over infected-untreated control suggested that these rhizobacteria can also indirectly suppress the nematode reproduction through ISR of tomato, this suggestion was supported by the adverse effect of such component tested, and especially Heat-killed cells which cloud inhibited the nematode reproduction. Results from these studies should contribute to a better understanding of the complex interactions among root-knot nematodes, introduced rhizobacteria and host plant. Such information would be valuable for the isolation and characterization of the active nematicidal agents or inducers agent or double impacts organisms. Also improving the performance of different bacterium by many procedures must be considered. However, to better use these isolates, more research is needed to determine their exact mode of action against nematodes on different hosts, their survival in soil, and efficient formulation and application methods.

\section{References}

Ali, N.I.; I.A. Siddiqui; S. S. Shaukat and M.J. Zaki (2002). Nematicidal activity of some strains of Pseudomonas spp. Soil biology \& Biochemistry, (34) 10511058.

Bruce, R.J. and C.A. West (1989). Elicitation of Lignin Biosynthesis and Isoperoxidase Activity by Pectic Fragments in Suspension Cultures of Castor Bean.Plant Physiology, (91) 889-897.

Buchenauer, H. (1998). Biological control of soil-borne diseases by rhizobacteria. Z. Pflanzenkrankh. Pflanzenschutz, (105): 329-348. 
Buonario, R. and P. Montalbini (1993). Peroxidase, superoxide dismutase and catalase activities in tobacco plants protected against Erysiphe cichoracearum by a necrotic strain of potato virus Y. Review of Patologia Vegetale, (3): 23-31.

Clark, R.S.; J. Kuc; R.E. Henze and F.W. Quacenbush (1959).The nature and fungitoxicity of an amino acid addition product of chlorogenic acid. Phytopathology, (49): 594-598.

Courath, U.; Z. X. Chen; J. R. Ricigliano and D. F. Klessig (1995). Two inducers of plant defense responses, 2,6-dichloroisonicotinic acid and salicylic acid, inhibit catalase activity in tobacco. Proc. Nat. Acad. Sci. USA, (92), 71437147.

Daniel, H.D. and C.M. George (1972). Peach seed dormancy in relation to endogenous inhibitors and applied growth substances J. Amer. Soc. Hort. Sci., (97): 651-654.

Giannakou, I.O.; I.A. Anastasiadis; S.R. Gowen and D.A. ProphetouAthanasiadou (2007). Effects of a non-chemical nematicide combined with soil solarization for the control of root-knot nematodes. Crop Protection, (26):1644-1654.

Hasky-Günther, K.; S. Hoffmann-Hergarten and R.A. Sikora (1998). Resistance against the potato cyst nematode Globodera pallid systemically induced by the Rhizobacteria Agrobacterium radiobacter (G12) and Bacillus sphaericus(B43). Fundam. Appl. Nematol., (21):511-517

Hayashi, M.; K. Wada and K. Munakata (1983). Synthesisand nematicidal activity of phenoxypropionic acid derivatives. Agric. Biol. Chem., (47): 2653-2655.

Hoffmann-Hergarten, S.; K. Hasky-Günther; M. Reitz, and R.A. Sikora (1997). Induced systemic resistance by rhizobacteria toward the cyst nematode Globodera pallidon potato, p. 292-295.In A. Ogoshi, K. Kobayashi, Y. Homma, F. Kodama, N. Kondo, and S. Akino (ed.), Plant growth-promoting rhizobacteria-present status and future prospects. Nakanishi Printing, Sapporo, Japan.

Holland, M.A. (1997). CAM's razor applied to hormonology: Are cytokinnins produced by plants. Plant Physiology, (115): 865-868.

Kerry, B.R. (2000). Rhizosphere interactions and exploitation of microbial agents for the biological control of plant-parasitic nematodes. Annu Rev Phytopathol, (38): 423-441.

Lebeda, A.; E. Kristkova and K. Dolezal (1999). Peroxidase isozyme polymorphism in Cucurbitapepo cultivars with various morphotypes and different level of field resistance to powdery mildew. Scientia Horticulturae, (81):103-112. 
Lotan, R.N. Sharon and D. Mirelman (1975). Interaction of the wheat germ agglutinin with bacteria cells and cell wall polmers. Eur. J. Biochem. (55), 257-262.

Mahajan, R.; P. Singh and K.L. Bajaj (1985). Nematicidal activities of some phenolic compounds against Meloidogyne incognita. Revue Nematology, (8): 161-164.

Mahdy, M.E. (2002). Biological control of plant parasitic nematodes with antagonistic bacteria on different host plants. PhD thesis (2002), pp.171.Hohen Landwirtschaftlichen Fakultät der Rheinischen FriedrichWilhelms- Universitätzu Bonn.

Marleny, Burkett-Cadena; Nancy, Kokalis-Burelle; Kathy S. Lawrence, Edzard van Santen and W.J. Kloepper (2008). Suppressiveness of root-knot nematodes mediated by rhizobacteria. Biological Control, (47), 55-59.

Maxwell, D.P. and F.D. Bateman (1967). Changes in the activities of some oxidases in extract of Rhizoctonia- infected bean hypocotyls in relation to lesion maturation. Phytopath., (57): 132.

Mori, M.; S. Hyeon; Y. Kimura and A. Susuki (1982). The nematicidal activity of acetylene compounds. Agtic. Biol. Chenu, (46):309-311.

Passardi, F.; C. Penel and C. Dun and (2004). Performing the paradoxical: How plant peroxidases modify the cell wall. Trends in Plant Science, (9):534-40.

Ramamoorthy, V.; R. Viswanathan; T. Raguchander; V. Prakasam and R. Samiyappan (2001). Induction by systemic resistance by plant growth promoting rhizobacteria in crop plants against pests and diseases. Crop Protection, (20): 1-11.

Reitz, M.; K. Rudolph; I. Schröder; S. Hoffmann-Hergarten; J. Hallmann and R. A. Sikora (2000). Lipopolysaccharides of Rhizobium etli strain G12 act in potato roots as an inducing agent of systemic resistance to infection by the cyst nematode Globoderapallida. Appl. Environ. Microbiol., (66): 35153518.

Reitz, M.; S. Hoffmann-Hergarten; J. Hallmann and R. A. Sikora (2001). Induction of systemic resistance in potato by rhizobacterium Rhizobium etli strain G12 is not associated with accumulation of pathogenesis-related proteins and enhanced lignin biosynthesis. Z. PflKrankh. PfISchutz., (108): 11-20.

Repka, V. and L. Lovakova (1994). Purification, characterization and accumulation of three virus induced cucumber peroxidases. Biologia Plantarum, (36):121132. 
Ryu, C.M.; M.A. Hu Farag; C.H. Reddy; M.S. Wei; H.X.Pare'; P.W. and J.W. Kloepper (2003). Bacterial volatiles promote growth in Arabidopsis. Proceedings of the National Academy of Sciences of the United States of America, (100): 4927-4932.

Schonbeck, F.; H.W. Dehne and W. Beicht (1980). untersuchungen zur aktivierung unspezifische resistenzmechanismen in Pflanzen. Z. PjlK. PfISChulz, (87):654-666.

Siddiqui, I.A. and S.S. Shaukat (2002). Rhizobacteria mediated Induction of Systemic Resistance (ISR) in Tomato against Meloidogyne javanica. Journal of Phytopathology, (150): 469-473.

Siddiqui, I.A. and S.S. Shaukat (2003). Suppression of root-knot disease by Pseudomonas fluorescens $\mathrm{CHAO}$ in tomato: importance of bacterial secondary metabolite,2,4-diacetylpholoroglucinol. Soil Biology \& Biochemistry, (35):1615-1623.

Siddiqui, I.A. and S.S. Shaukat (2004). Systemic Resistance in Tomato Induced by Biocontrol Bacteria Against the Root-KnotNematode, Meloidogynejavanica is Independent of Salicylic Acid Production. J. Phytopathology, (152): 48-54.

Siddiqui, I.A. and S.S. Shaukat (2005). Pseudomonas aeruginosa- mediated Induction of Systemic Resistance in Tomato Against Root-knot Nematode. Plant Pathology Journal 4 (1): 21-25.

Siddiqui, Z.A. and I. Mahmood (1999). Role of bacteria in the management of plant parasitic nematodes: a review. BioresourceTechnol, (69): 167-179.

Siddiqui, Z.A; G. Baghel and M.S. Akhtar (2007). Biocontrol of Meloidogynejavanica by Rhizobium and plant growth-promoting rhizobacteria on lentil. World J Microbiol Biotechnol, (23): 435-441.

Sridhar, R. and S.H. Ou (1974). Biochemical changes associated with development of resistant and susceptible types of rice blast lesion. Phytopathol. Z., (79): 222-230.

Valerie, N. K.; A.D. Kerrie and S.S. Eileen (2001).Chemical and biological induction of resistance to the clover cyst nematode (Heteroderatrifolii) in white clover (Trifolium repens). Nematology, (3): 35-43.

Van Loon, L.C. and P.A.H.M. Bakker (2005).Induced systemic resistance as a mechanism of disease suppression by rhizobacteria. In Z. A. Siddiqui (Ed.), PGPR: Biocontrol and biofertilization (pp. 39-66). Dordrecht, The Netherlands: Springer Science. 
Van Loon, L.C.; P.A.H.M. Bakker and C.M.J. Pieterse (1998). Systemic Resistance Induced by Rhizosphere Bacteria. Annu. Rev. Phytopathol., (36): 453-83.

Veech, J.A. (1979). Histopathological localization and nematoxicity of terpenoid aldehydes in cotton. J. Nernatol., (11): 240-246.

Vetrivelkalai, P.; M. Sivakumar and E.I. Jonathan (2010). Biocontrol potential of endophytic bacteria on Meloidogyne incognita and its effect on plant growth in bhendi. Journal of Biopesticides, 3(2): 452-457. 


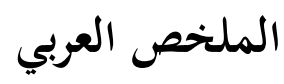

تقييم بعض أنواع بكتريا التربة كمستحثات لمقاومة نباتات الطماطم أو كعوامل حيوية لمكافحة نيماتودا تعقد الجذور (ميلودوجيني انكوجنيتا)

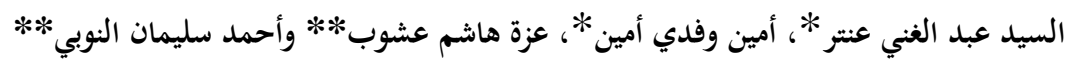
* *قسم الحيوان والنيماتولوجيا الزراعية- كلية الزراعة- جامعة القاهرة.

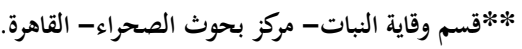

بعد مجموعة من تجارب التقييم في المعمل و الصوبة للعديد من بكتريا التربة المعزولة من منطقة

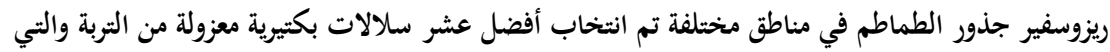

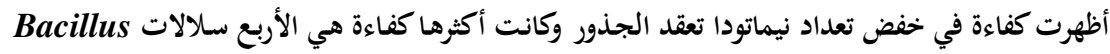
brevis, Bacillus cereus, Methylomonas methanica and Obesumbacterium

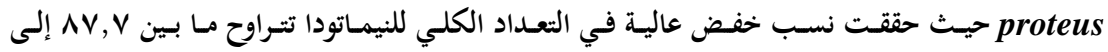

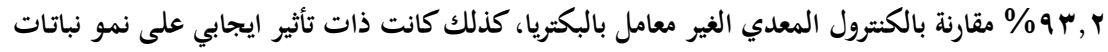

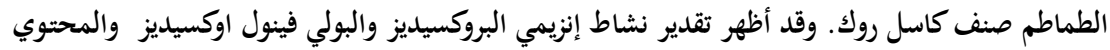

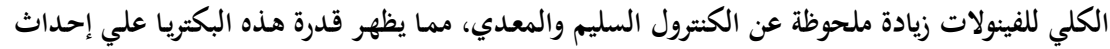
تغييرات بيوكيميائية داخل نبات الطماطم وتعتبر دلائل على استحثاث مقاومة النبات ضد الد النيماتودا.

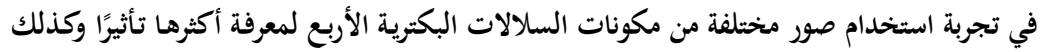

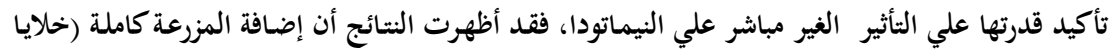

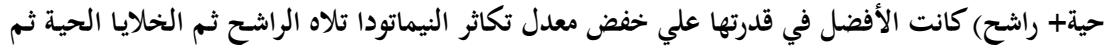
الخلايا الميتة. مما يشير إلى قدرة هذه السلالات البكتيرية علي تثبيط معدل تكاثر النيماتودا من خحلال تنشيط مقاومة النبات. يستنتج من هذه النتائج أن تلك السلالات من الريزوبكتريا استطاعت أن تحد من تكاثر النيماتودا من خحلال قدرتها على استحثاث المقاومة لنباتات الطماطم، ويقترح إجراء المزيد من التجارب الحقلية عليها وتقييم كفاءتها على أنواع نباتية أخرى لإدخالها في برامج مكافحة النيماتودا. 